\title{
Candida auris fungemia in a non-diabetic COVID-19 positive patient-An unusual case report.
}

\author{
Malhotra $\mathrm{S}^{2 *}$, Venugopal $\mathrm{A}^{2}$, Sinha $\mathrm{KK}^{2}$, Bhatia $\mathrm{NJK}^{2}$, Kaur A ${ }^{2}$, Duggal $\mathbf{N}^{2}$, Varshney $\mathrm{AK}^{1}$ \\ ${ }^{1}$ Department of General Medicine, ABVIMS \&Dr. RML Hospital, New Delhi, India. \\ ${ }^{2}$ Department of Microbiology, ABVIMS \&Dr. RML Hospital, New Delhi, India. \\ *Corresponding Author: Malhotra S, Department of General Medicine, ABVIMS \& Dr. RML Hospital, New Delhi, India.
}

Received date: 02 October 2021; Accepted date: 14 October 2021; Published date: 16 October 2021

Citation: Malhotra S, Venugopal A, Sinha KK, Bhatia NJK, Kaur A, et al. (2021) Candida auris fungemia in a non-diabetic COVID-19 positive patient-An unusual case report. J Med Case Rep Case Series 2(11): https://doi.org/10.38207/JMCRCS/2021/0211174

Copyright: (C) 2021 Malhotra S. This is an open-access article distributed under the terms of the Creative Commons Attribution License, which permits unrestricted use, distribution, and reproduction in any medium, provided the original author and source are credited.

\begin{abstract}
Candida Auris (C. Auris) is an emerging yeast that can present as a global health threat. It has the capacity to create havoc in patients specially admitted in ICUs. Rapid spread from patient to patient in a hospital setting and multidrug resistance leads to nosocomial outbreaks. Diagnostic microbiology laboratories are generally unable to identify it due to a lack of expertise and infrastructure. Hospital infection prevention and control measures are the only possible means to cut the chain of nosocomial transmission. Here we report the first case of C.auris septicaemia in a postCOVID non-diabetic patient.
\end{abstract}

Keywords: Candida Auris fungemia, ICU, hospital-acquired infection.

\section{Introduction}

The threat of fungal infection has been increasing in recent times which is a notable case of concern. About 1.7 billion people worldwide suffer from fungal infections. [1] The predominant cause of nosocomial infection is found to be Candida species which is also the fourth leading cause of all hospital-acquired infections. Annually, there are approximately 400,000 bloodstream infections caused by Candida species globally, with mortality rates exceeding $40 \%$. [1] Candida Auris (C. Auris) is an emerging multidrug-resistant nosocomial pathogen likely to cause outbreaks in hospitals especially in the ICU settings. It was first isolated in the ear discharge of a Japanese female patient in Tokyo in 2009. In recent years, the Centre for Disease Control and Prevention (CDC), Atlanta, USA has released a clinical alert to healthcare facilities warning of the international emergence of $C$. Auris infections with high mortality rates.[2]

\section{Case Presentation}

A 62-year-old male presented in the emergency department of our hospital with the chief complaints of fever, cough, generalized weakness, decreased appetite, and altered sensorium for 2 days. He was a known case of seizure disorder. On examination, he was found to be hypotensive $(\mathrm{BP}=90 / 60 \mathrm{mmHg})$ and tachypnoeic $(\mathrm{RR}=32 \mathrm{bpm})$. He was started on inotropic support and bi-level positive airway pressure (BIPAP). CT chest showed features of COVID pneumonia. In view of the COVID 19 positive report by RT-PCR, he was shifted to COVID ICU and was started on antibiotics (in. piperacillin+tazobactam-4.5G QID and in. teicoplanin- OD), anti-epileptics (levetiracetam 1g IV BD), and other supportive medications. The endotracheal aspirate culture showed growth of Acinetobacter species and blood culture showed growth of Klebsiella species5 days post ICU admission. Due to
C. Auris exhibits a great concern due to difficulty in identification with traditional methods in laboratories, multidrug-resistant patterns, and person-to-person transmission. The virulence pattern is the same as that of other candida species, but it also can evade innate immunity and form biofilms making it resistant to almost all antifungal agents.[3] The emergence of severe acute respiratory syndrome coronavirus 2 (SARS-CoV-2) in late 2019 has caused a pandemic of COVID-19.[4] Various respiratory supports ranging from the nasal cannula to invasive ventilation in the affected patients has bought attention to the secondary transmission of multidrug-resistant organisms including. Auris in COVID-19 ICUs.COVID-19 patients who are pre-colonized with C. Auris and require indwelling devices such as central venous and Foley's catheters have higher risks of the development of C. Auris bloodstream and urinary tract infections.[5]

worsening hypoxia, he was started on mechanical ventilation, and a tracheostomy was done eventually. Patient's COVID status became negative after 25 days of admission, but his general condition was not improving. Antibiotic escalation (in. meropenem 1gm TDS) was done according to culture sensitivity reports of subsequent samples (blood $\mathrm{c} / \mathrm{s}$ and ET aspirate c/s). Follow-up chest X-RAY showed pleural effusion and pneumatocele. On the 35th-day post ICU admission, blood and urine cultures were sent again. The blood culture bottle showed a signal in 18 hours and the subculture on $5 \%$ sheep blood agar (SBA) (HI Media, Mumbai) showed the growth of yeast-like colonies. Gram staining of these colonies showed budding yeast-like cells. Subculture was made on Sabouraud Dextrose Agar (HI Media, Mumbai) and creamy, pasty colonies were obtained after 48 hours of incubation 
at $37^{\circ} \mathrm{C}$ suggesting growth of Candida spp. Subculture on Chrom agar showed pinkish colour colonies after 48 hours of incubation at $37{ }^{\circ} \mathrm{C}$ (HiCrome ${ }^{\mathrm{TM}}$ HiMedia, Mumbai).

VITEK 2 compact (bioMérieuxInc., Durham, USA) system identified the isolate as Candida Auris with $99 \%$ probability. The Antifungal susceptibility testing by both disc diffusion and MIC (E-strip HiMedia, Mumbai) method showed resistance to fluconazole and voriconazole. MIC of fluconazole and voriconazole was 96 and 8 respectively. A semi-quantitative urine culture on $5 \% \mathrm{SBA}$ also showed growth of Candida spp. with a count of $>=10^{5} \mathrm{CFU} / \mathrm{ml}$ was identified as C.auris by VITEK 2 compact (bioMérieux Inc., Durham, USA) system with the same probability.

Based on the above findings, the patient was immediately put on IVcaspofungin 50mg for 5 days.

\section{Discussion}

Since 2019, the rapid spread of novel coronavirus has led to the complete transformation of healthcare facilities into dedicated COVID care centers, and tremendous pressure is infused on hospitals to accommodate the constantly increasing cases of COVID-19 patients. This in turn has led to the suboptimal application of infection control practices which in turn has become a ground for the spread of nosocomial infections. C. Auris has already emerged as a multidrugresistant fungal pathogen globally with a great potential for nosocomial transmission. A report from Oman in the year 2021 has shown that there is an increased risk for Candida spp. super-infection in COVID-19 patients, resulting in poor patient outcomes.[6]

Here, we report the first case of $C$. Auris fungemia in a nondiabetic patient with an underlying risk factor of prolonged hospitalization and post COVID-19 severe pneumonia on broad-spectrum antibiotics and respiratory support. Previously there are reports of C.auris infections in COVID-positive patients both from India and abroad but all these patients were diabetic, unlike our case where the patient was nondiabetic.[1,4,5,7]

Accurate identification of this yeast is possible by DNA sequencing or MALDI-TOF. Most of the Laboratories lack these facilities and hence timely identification is not possible leading to sub-optimal treatment. CDC(Atlanta, USA) has issued guidelines for the management of $C$. Auris infection but compliance to these guidelines in resource-poor

\section{Conclusion}

This is the first case report of C.auris from a non-diabetic post COVID patient from our hospital which has put some insight on this yeast that it can cause infection in post-COVID patients. Any multi-drug resistant Candida species isolated from a critically ill patient with underlying morbidities, which has been difficult to identify by the

Acknowledgment: We acknowledge Ms. Neetu Singh (MTS) for all sample processing and record maintenance.
Journal of Medical Case Reports and Case Series ${ }^{-}$ISSN: 2692-9880 Infection control practices were restrengthened and surveillance for $C$. Auris was started as per CDC (Atlanta, USA) guidelines (swabs from axilla and groin from all patients admitted at that time $\&$ for each new patient admitted till one month time from the date when the first case isolated). Chlorhexidine-based oral hygiene /body sponging practices were complied with. Cleaning and disinfection practices for instruments and surfaces were also monitored stringently

On the $5^{\text {th }}$ day of starting the antifungal, the patient started developing hyperthermia which was continuous and there was worsening of Glasgow coma scale (GCS). Repeated blood \& urine cultures were sent thrice and similar culture reports showing growth of C.auris were obtained. However, on day 36 patient succumbed to his illness of postCOVID pneumonia, C. Auris septicemia with ARDSwith superimposed bacterial pneumonia, and shock with the cause of death attributed to respiratory failure.

settings is difficult as it involves surveillance of all patients in facilities that have had new cases of $C$. Auris and identifying the species of all Candida isolates from any specimen source (normally sterile and non-sterile sites) for at least 1 month until there is no evidence of $C$. Auris transmission along with a screening of these patients for C.auris carriage by taking composite swabs from axilla and groin. Other infection prevention and control practices like hand hygiene adherence, barrier nursing leading to transmission-based precautions and cleaning and disinfecting the patient care environment (daily and terminal cleaning), and reusable equipment with recommended products also need to be reinforced.[8]

Our results have shown resistance to fluconazole and voriconazole with high MIC. A study from middle- eastern countries has shown low MIC to echinocandins and high MIC to fluconazole in whereas MIC of Amphotericin B varied among cases.[9]

According to the $\mathrm{CDC}$, institutions should investigate the possibility of an outbreak even when a single case has been identified.[8] Thus surveillance should be mandatory when a patient is diagnosed with a bug. Adequate infection prevention and control practices should be implemented to control the outbreak, transmission, and mortality rates among the patients.[10]

conventional diagnostic methods, should raise the suspicion of the possibility of C.auris infection. Rapid detection and reporting play a vital role in the prevention of outbreak situations. A strict and effective infection control practice including patient isolation, barrier nursing, and strict hand hygiene practice is necessary to cut off the transmission.

Financial Support and Sponsorship: Nil.

Conflicts of Interest: There are no conflicts of interest. 


\section{References}

1. Bhat K S, Kisku KH, Kanungo R (2019) Candida auris candidemia an emerging threat: A case report and mini review of literature. $\mathrm{J}$ Curr Res Sci Med. 5(2): 110-3.

2. Cortegiani A, Misseri G, Fasciana T, Giammanco A, Giarratano A, et al. (2018) Epidemiology, clinical characteristics, resistance, and treatment of infections by Candida auris. $\mathrm{j}$ intensive care. 6: 69.

3. Lone SA, Ahmad A (2019) Candida auris - the growing menace to global health. Mycoses. 62(8): 620-637.

4. Allaw F, Kara Zahreddine N, Ibrahim A, Tannous J, Taleb H, et al. (2021) First Candida auris Outbreak during a COVID-19 Pandemic in a Tertiary-Care Center in Lebanon. Pathogens. 10(2): 157.

5. Chowdhary A, Sharma A (2020) The lurking scourge of multidrug resistant Candida auris in times of COVID-19 pandemic. J Glob Antimicrob Resist. 22: 175-176.
6. Al-Hatmi AM, Mohsin J, Al-Huraizi A, Khamis F (2020) Covid-19 associated invasive candidiasis. J Infect. 82(2): e45-e46.

7. Villanueva-Lozano $\mathrm{H}$, Treviño-Rangel RJ, González GM, Ramírez-Elizondo MT, Lara-Medrano R, et al. (2021) Outbreak of Candida auris infection in a COVID-19 hospital in Mexico. Clin Microbial Infect. 27(5): 813-816.

8. CDC guidelines on Infection Prevention and Control for Candida auris.July 192021.

9. Chatterjee S, Alampalli SV, Nageshan RK, Chettiar ST, Joshi S, et al. (2015) Draft genome of a commonly misdiagnosed multidrug resistant pathogen Candida auris. BMC Genomics. 16(1): 686.

10. Biswal M, Rudramurthy SM, Jain N, Shamanth AS, Sharma D, et al. (2017) Controlling a possible outbreak of Candida auris infection: Lessons learnt from multiple interventions. J. Hosp. Infect. 97(4): 363-370. 